\title{
Area-specific self-regulation of slow cortical potentials on the sagittal midline and its effects on behavior *
}

\author{
Niels Birbaumer ${ }^{a, b}$, Larry E. Roberts ${ }^{c}$, Werner Lutzenberger ${ }^{a}$, Brigitte Rockstroh ${ }^{d}$ \\ and Thomas Elbert ${ }^{e}$ \\ a Department of Psychology, University of Tübingen, D7400 Tübingen (F.R.G.), ' Dipartimento di Psicologia Generale, Università degli Studi, Padua \\ (Italy), ' Department of Psychology, McMaster University, Hamilton, Ont. L8S 4K1 (Canada), 'Department of Psychology, University of Konstanz, \\ D7750 Konstanz (F.R.G.), and ${ }^{e}$ Institute of Experimental Audiology, University of Münster, D4400 Münster (F.R.G.)
}

\begin{abstract}
Summary Exteroceptive feedback was given for negative and positive shifts in slow potentials ( $\mathrm{SPs}$ ) recorded from $\mathrm{Fz}, \mathrm{Cz}$, or $\mathrm{Pz}$ (between groups design). Slow potentials at the feedback site were referred to adjacent scalp and non-cephalic electrodes, so as to confine SP shifts to the feedback location. Area-specific regulation of SPs was obtained at each midsagittal site after 3 days of feedback training. Subjects. reported sensorimotor and emotional arousal when negative SP shifts were trained frontally, but not when negative shifts were trained parietally (cognitive/attentional strategies reported after parietal feedback). Area-specific regulation of SPs was subsequently abolished when behavioral tasks were added to further probe frontal/parietal differences (dual-1ask procedure). These findings indicate that area-specific self-regulation of SPs is possible on the sagittal midline, and that self-regulated parietal SPs (in contrast to frontal ones) arise from non-motoric generators. The source of SP self-regulation was more readily probed by verbal reports of feedback strategy than by study of dual-task relations, because feedback control was disrupted by the dual-task requirement.
\end{abstract}

Key words: Slow cortical potentials; Feedback learning; Stimulus preceding negativities; Threshold regulation of EEG

Previous research has shown that human subjects can be trained to modify surface-recorded slow cortical potentials (SPs) on command, when exteroceptive feedback is provided for these potentials (Rockstroh et al. 1984). Self-control has been extensively documented for the case in which feedback is given for negative and positive SPs recorded at the vertex (Birbaumer et al. 1980) and more recently for the case in which feedback is made conditional on the differential polarization of the hemispheres between left-central (C3) and rightcentral (C4) recording electrodes (Birbaumer et al. 1988; Rockstroh et al. 1990a). Feedback regulation of SPs is of interest, because study of the behavioral effects of such regulation provides information about the composition and role of SPs in behavior generally (Birbaumer et al. 1990).

SPs that are self-regulated at frontal and central locations appear to reflect the known sensorimotor

Correspondence to: Larry E. Roberts, Department of Psychology, McMaster University, Hamilton, Ont. L8S 4K1 (Canada).

* This research was supported by the Deutsche Forschungsgemeinschaft (S.F.B. 307) and the NATO Division of Scientific Affairs $(87 / 0503)$. functions of cortical tissues proximal to these recording sites. Sensorimotor tasks such as reaction time, haptic discrimination, and self-initiated button pressing are facilitated when negative SPs are induced by feedback at the vertex $(\mathrm{Cz})$, compared SP positivity induced at this site (see Rockstroh et al. 1989, for a review). Human subjects report having used muscle tension and/or movement preparation when vertex negativity is called for, but not when vertex positivity is trained (Roberts et al. 1989). Modulation of contralateral hand preference by SP negativity has also been demonstrated when feedback is given for the SP difference between left and right central electrodes (Rockstroh et al. 1990b). These effects of feedback-induced SP modulation on sensorimotor functions are consistent with slow wave phenomena that occur when motor behavior is induced by explicit experimental manipulation. For example, slow negativities are known to accompany motor responses on reaction time tasks and to depend on the speed and force with which such responses are performed (Gaillard 1986; Coles 1989). Unsignalled voluntary movements are also preceded by a slow negativity (the "readiness potential") that commences 1 or $2 \mathrm{sec}$ before movement is observed. Slow negativities produced by feedback training and self-initiated movement are more pronounced at central and frontal sites 
where motor functions are represented than at other ;calp locations (Roberts et al. 1989; Chwilla and Brujia 1991).

Although the evidence just reviewed points to an nfluence of motoric functions on SP negativity -ecorded at frontocentral sites, it does not necessarily ollow that slow negativities at these or other sites arise only from motoric generators. Several researchers (e.g., Tecce 1972; Elbert and Rockstroh 1987; Birbaumer et il. 1990) have suggested that SP negativity reflects a nore general neurophysiological mechanism that presares neural networks for highly specific information srocessing, and that SPs thus generated may or may ot involve a motor component, depending on the task applied. This hypothesis is consistent with the early esearch of Weinberg (1972), who showed that negative ;low waves occurred in the absence of movement when subjects anticipated the delivery of stimuli containing nformation about the correctness of a just-completed notor response. Subsequent research has confirmed hat these "stimulus preceding negativities" (i) can be separated from movement-related potentials (Damen and Brunia 1987, 1991), (ii) increase with the informaion content of the anticipated stimulus (Ruchkin et al. 1986; Chwilla and Brunia 1991), and (iii) distribute opographically in accordance with the sensory modalty within which the stimulus is delivered (central domirance when kinesthetic pathways are utilized, and a nore posterior focus when the informational cue is sresented in vision; see Rösler and Heil 1991). This zvidence for slow negativities occurring in the absence of motor behavior raises the possibility that SPs can be tissociated from motoric processes through feedback methods, particularly if SP shifts are restricted to neuronal networks posterior to central motor areas. Howzver, at present we do not know whether SPs can be self-regulated in an area-specific fashion in these areas and, if so, how behavioral performance is affected.

The experiment reported in this article was conzerned with the latter two questions. One goal was to determine whether area-specific SP shifts could be induced at different locations on the sagittal midline when subjects were given exteroceptive feedback for those shifts. Another goal was to contrast area-specific precentral and postcentral SP self-regulation with regard to their dependence on motoric functions. To achieve these objectives, separate groups of subjects wère given exteroceptive feedback for modulating their SPs in an area-specific fashion at frontal $(\mathrm{Fz})$ or parietal $\left(\mathrm{P}_{\mathrm{z}}\right)$ electrodes. A third group received feedback for SPs confined to the vertex $(\mathrm{Cz})$, to provide a further reference point for assessing area-specific effects. After 3 days of area-specific feedback training, we probed the basis of SP regulation by superimposing on the feedback procedure behavioral tasks that were believed to differentially tap motoric and non-motoric functions. We also assessed the basis of SP regulation by analyzing verbal reports that subjects gave of their SP control strategies. To ensure the validity of the verbal report data, we used a feedback procedure that allowed self-report to arise only from the subject's memory of behavioral events related to feedback, and not from other sources of self-report that may operate in a feedback situation (e.g., response bias induced by task instructions or spatial orientation of the feedback display; see Roberts et al. 1984). We were particularly interested in a contrast of frontal and parietal areaspecific SP self-regulation. A provisional hypothesis was that motoric functions would be implicated when SPs were regulated at the frontal midline, but not at the parietal midline site.

\section{Method}

\section{Subjects}

Eighteen student volunteers ( 9 males, 9 females, age 20-35 years) were randomly assigned to the frontal, central, and parietal groups, with the restriction that sample size be equal in the 3 conditions. Subjects were free of medication and central nervous system or cardiovascular abnormality. Subjects were paid 20 Deutschmarks (about \$12 U.S.) per session plus bonus money of approximately 10 Deutschmarks per session according to their success on the feedback task.

\section{Apparatus and physiological recordings}

The electroencephalogram (EEG) was recorded monopolarly at $\mathrm{Fpz}, \mathrm{Fz}, \mathrm{Cz}, \mathrm{Pz}$, and $\mathrm{Oz}$ following the 10-20 system. Electrodes were affixed to the ear lobes, 2nd vertebra, and right clavicle to serve as non-cephalic reference points. The skin at each site was prepared by cleaning with alcohol and applying an abrasive paste (Omniprep). Non-polarizing $\mathrm{Ag} / \mathrm{AgCl}$ electrodes (Zack) were used. The EEG was amplified by a Nihon-Kohden amplifier specially manufactured to have a time constant of $10 \mathrm{sec}$. Signals were sampled at $100 \mathrm{~Hz}$. Amplifier output was filtered by the method of Elbert and Rockstroh (1980) to give the DC component. We will refer to the filtered EEG channels as SPs.

Electro-oculograms (EOGs) consequent on vertical (VEOG) and lateral (LEOG) eye movements were also recorded at $100 \mathrm{~Hz} . \mathrm{Ag} / \mathrm{AgCl}$ electrodes were affixed $1 \mathrm{~cm}$ above and below the left eye and at the outer canthi. These measurements were used to prevent contamination of SPs by eye movement artifact (see below).

A network of computers (PDP $11 / 73$ and 8/e) was used to generate the experimental stimuli, implement the feedback contingencies, and store digitized physiological data. 


\section{Feedback procedure}

Continuous visual feedback was provided for SP shifts occurring on trials of $8 \mathrm{sec}$ duration. The feedback stimulus was the outline of a rocket ship that appeared on a $30 \times 40 \mathrm{~cm}$ television screen situated 2 $\mathrm{m}$ in front of the subject at eye level. The rocket moved back and forth in a horizontal plane through a gap formed by an upper and lower vertical bar (these bars situated slightly to the left of center on the screen). Trials were designated by presentation of the rocket in the central gap, together with a discriminative stimulus (the letter " $A$ " or " $B$ ") at the right boundary of the display. On each trial the subject's task was to move the rocket out of the gap toward the discriminative stimulus situated at the right boundary of the screen. Excursions of the rocket in the opposite direction (error feedback) were discouraged by the instructions that subjects received. Assignment of the SP responses (negativity/positivity) to the discriminative stimuli (the letters $\mathrm{A}$ and $\mathrm{B}$ ) varied randomly between subjects. It should be noted that correct responding moved the rocket toward the right, and incorrect responding to the left, on both the A and B trial types. Subjects therefore received the same performance instructions and attempted the same (right ward) feedback excursions on $\mathrm{A}$ and $\mathrm{B}$ trials, but the $\mathrm{SP}$ responses (negativity/positivity) that produced these excursions were different between the two trial types (within-subject discriminative operant procedure, after Roberts et al. 1989). Subjects were not informed of the responses that were trained, but relied instead on feedback as a guide to success.

In order to avoid the cortical evoked response elicited by visual stimulation, the position of the rocket remained fixed at the central gap for the first second of each feedback trial. For the remainder of the trial ( $\mathrm{sec}$ 2-8) movements of the rocket were a linear function of the integrated EEG referred to the mean of a $4 \mathrm{sec}$ pretrial baseline (SPs). Subjects in the frontal, central, and parietal groups received feedback for SP shifts recorded at $\mathrm{Fz}, \mathrm{Cz}$, and $\mathrm{Pz}$, respectively. In order to increase the likelihood of area-specific regulation in these groups, the reference point for measurement of SPs included not only the ear lobe and non-cephalic sites, but also scalp locations adjacent to the site at which specificity was sought. Thus, in the frontal group SPs recorded at $\mathrm{Fz}$ were referred to an average of recordings taken at $\mathrm{Fpz}, \mathrm{Cz}, \mathrm{Pz}, \mathrm{Oz}$, the linked ear lobes, and the non-cephalic electrodes. Similarly, in the central group feedback was given for the $\mathrm{Cz}$ recording referenced to the average of all remaining sites, and in the parietal group for the $\mathrm{Pz}$ recording similarly referenced. Reference sites were linked by digital averaging and not by hard-wired contact. To prevent spurious EEG recordings due to vertical eye movements, a time-out contingency arrested excursions of the rocket toward the letters $A$ and $B$ whenever VEOGs of the same polarity as the required SP shifts were detected (for a detailed description of artifact control procedures, see Elbert et al. 1985).

Subjects received bonus money for performing successfully on the feedback task. Whenever the obtained $\mathrm{SP}$ shift exceeded either $-6 \mu \mathrm{V}$ or $+6 \mu \mathrm{V}$ from baseline, the outline of the rocket became filled, signalling that the subject had earned or lost a "win" point worth 2 Deutschmarks (depending on whether the rocket was on the success or failure side of the starting gap). Subjects also received transfer trials on which the letters A or B were presented but feedback was removed. Subjects were told that they should produce the required response " $A$ " or " $B$ " as best they could on these trials, even though feedback and win points would not be displayed to tell them how successful they had been.

Previous research has shown that stimulus properties inherent in a feedback display elicit a negative SP shift that is large at $\mathrm{Cz}$ compared to frontal $(\mathrm{Fz})$ and parietal ( $\mathbf{P z}$ ) sites (Birbaumer et al. 1980). If this elicited effect is not removed, SP negativity may be achieved at $\mathrm{Cz}$ with little effort by the subject, whereas production of SP positivity at this site will be more difficult Earlier studies of $\mathrm{Cz}$ feedback have therefore typically included a constant positive offset $(+6 \mu \mathrm{V})$ on all trials, to better equate the difficulty of the opposing trial types (e.g., Birbaumer et al. 1980; Roberts et al. 1989). However, this practice was of doubtful merit in the present study, in which feedback was given at frontal $(\mathrm{Fz})$ and parietal (Pz) locations where elicited effects consequent on the feedback display are less extreme. Because the frontal and parietal groups were of primary interest, we decided to remove the offset in all groups, so as to apply the same feedback method to each condition. We accepted that self-regulation by the central group might be affected by this modification, but comparison of our results with earlier findings was possible (Roberts et al. 1989) and provided information on the question.

Subjects received 3 sessions of feedback training scheduled on consecutive days. Each session commenced with a block of 30 feedback trials (15 A and 15 B) followed by a block of 20 transfer trials ( 10 of each kind). This-sequence was then repeated once to give a total of 100 trials for the session. Within blocks, A and $\mathrm{B}$ trials were given in a mixed order. The time lapsing from the end of one trial to commencement of the next pretrial baseline varied randomly between 4 and 13 sec.

\section{Behavioral tasks}

In the fourth (last) session of the experiment, behavioral tasks were added to the feedback procedure, which continued as before. As a "frontal" task, sub- 
jects were asked to press a button with their right hand at intervals of their choosing, while self-regulating their SPs. This task was imposed only on transfer trials. On the basis of previous evidence indicating facilitation of sensorimotor performance by frontal negativity (Stamm 1984; Birbaumer et al. 1990), it was hypothesized that subjects in the frontal group would press the button more often when negativity was required at the frontal electrode than when positivity was required at this site. However, performance on this task was not expected to differ between negativity and positivity in subjects trained to self-regulate at the parietal location.

A different task was used to tap parietal functions. On this task a series of common objects was added to the feedback display on each feedback trial. Different objects were given for $\mathrm{A}$ and $\mathrm{B}$ trials, but each object remained in a constant location over trials. Subjects received no instructions as to the significance of the objects. However, at the end of the training session, incidental memory for location was tested by asking subjects to position the objects correctly on the feedback screen. It was hypothesized that parietal subjects would remember spatial position more accurately when objects were presented during parietal negativity as compared to parietal positivity, and that this task would not differ as a function of negativity/positivity in the frontal group (Stamm 1984).

\section{Verbal reports}

Upon conclusion of the fourth session, subjects were asked without prior notification to describe what they had done over the course of feedback training to self-regulate their SPs on negativity and positivity trials. Subjects described their performance strategies by placing an " $A$ " and a " $B$ " on ordinal scales addressing each of the following activities ( $3=$ a great deal, $-3=$ the opposite pole; midpoint of $0=$ no change): (1) concentration versus distraction; (2) deep, fast breathing versus slow, shallow breathing; (3) muscle tension versus muscle relaxation; (4) concrete imagery versus no imagery; (5) prepared a rapid movement versus remained still; (6) imagined an unpleasant versus pleasant situation; (7) attended to the direction of the rocket versus no attention to the display; (8) emotional tension versus calm; (9) thought about strategy versus did not do so; (10) imagined objects in the room versus no attention to such objects; (11) suspended versus normal breathing; and (12) thought about objects on the screen versus no attention to these objects. On the basis of previous evidence (Rockstroh et al. 1982; Roberts et al. 1989; Elbert et al. 1992), we hypothesized that scales assessing sensorimotor functions $(2,3$, 5 and 11) would differentiate between SP negativity and positivity in the frontal and central groups. On the other hand, scales assessing attentional functions $(1,7$, 9,10 and 12) were hypothesized to differentiate be- tween negativity/positivity in the parietal group. An additional scale required that subjects rate their perceived success on A and B trials ( $3=$ very successful; $-3=$ not successful at all). ${ }^{1}$

\section{Data reduction}

Self-regulation of SPs was assessed by subtracting - the mean SP observed during the last second of the pretrial period from the mean observed during the last $7 \mathrm{sec}$ of the trial period (the interval during which feedback was provided). In order to be sure that SP modulation was achieved at the feedback electrode and not at one of the digitally averaged reference sites, these "change scores" were based on SPs referenced to linked ears only. ${ }^{2}$ Change scores were averaged separately for negativity and positivity trials in each feedback and transfer block, thus giving 8 summary measures per subject in each session ( 2 trial types $\times 2$ feedback blocks $\times 2$ transfer blocks) at each recording site. Trials on which the DC shift exceeded $100 \mu \mathrm{V}$ in one of the EEG channels or $30 \mu \mathrm{V}$ in either of the EOG channels were excluded from this analysis, and from the analysis of all other response measures. These calculations were performed for the third session of training (the last feedback-only session), and also for the fourth session on which the frontal and parietal tasks were added to the training procedure.

It was often convenient to summarize the effect of feedback training on response measures (SPs, task performance, and verbal reports) by subtracting $\mathrm{A}$ and $B$ trials from one another. Positivity trials were subtracted from negativity trials when this was done. Measures computed this way are referred to as "bidirectional."

The plan for statistical analyses was adopted from the study of Roberts et al. (1989) in which subjects were trained to regulate SPs area-non-specifically at the vertex. Unless otherwise stated, all tests were 2 tailed (1-tailed accepted only where directional predic tions were made). Greenhouse-Geisser corrections $(\epsilon)$ are reported where sphericity violations were found, and significance levels were adjusted accordingly.

\footnotetext{
Terbal report data were not available for one subject in each of the frontal and parietal groups. Sample size was reduced by one subject in these groups, when the verbal report data were analyzed.

2 The aigorithm used to compute exteroceptive feedback in this study (SP at the feedback site minus the average of 8 reference sites) favored area-specific manipulation of SPs at the feedback electrode over alternative manipulation of SPS at one or more reference sites, because SP shifts at the feedback electrode (the first term in the equation, weighted 1.0) had greater influence on the feedback calculation than did any reference site considered singly (each reference site contributing $1 / 8$ of the reference potential). The SP effects reported herein did not differ when compared between the two reference caiculations.
} 


\section{Results}

\section{Slow potentials}

Changes in SPs on negativity and positivity trials of the concluding feedback-only session are shown in Fig. 1 , for the frontal, central, and parietal groups separately. Slow potential shifts are given for the feedback site (diagonal, upper left to lower right) and the two remaining mid-sagittal locations, collapsed over all trials of this session. Inspection of Fig. 1 shows that each group differentiated their SPs in the direction of training primarily or exclusively at the feedback electrode. Bidirectional differences were significant (1-tailed) at $\mathrm{Fz}$ in the frontal group, $t(5)=2.49, P<0.05$, at $\mathrm{Pz}$ in the parietal group, $t(5)=2.69, P<0.025$, and at $\mathrm{Cz}$ in the central condition, $t(5)=2.97, P<0.025$ (in the latter case on feedback trials only). Bidirectional differences were not significant at any other recording site (including $\mathrm{Fpz}$ and $\mathrm{Oz}$ ) in the 3 feedback conditions $(t<1)$. Area specificity was corroborated by analysis of variance which revealed an interaction of Groups $x$ Negativity /Positivity $\times$ Electrode site, $F(4,30)=3.1$, $P<0.05, \epsilon=0.96$. Main effects and interactions attributable to Feedback/Transfer were not significant in this analysis, indicating comparable performance across these trial types.

The same analyses were repeated for the fourth session of the experiment, in which the behavioral tasks were added to the feedback procedure (dual-task requirement, objects for later repositioning on feed-
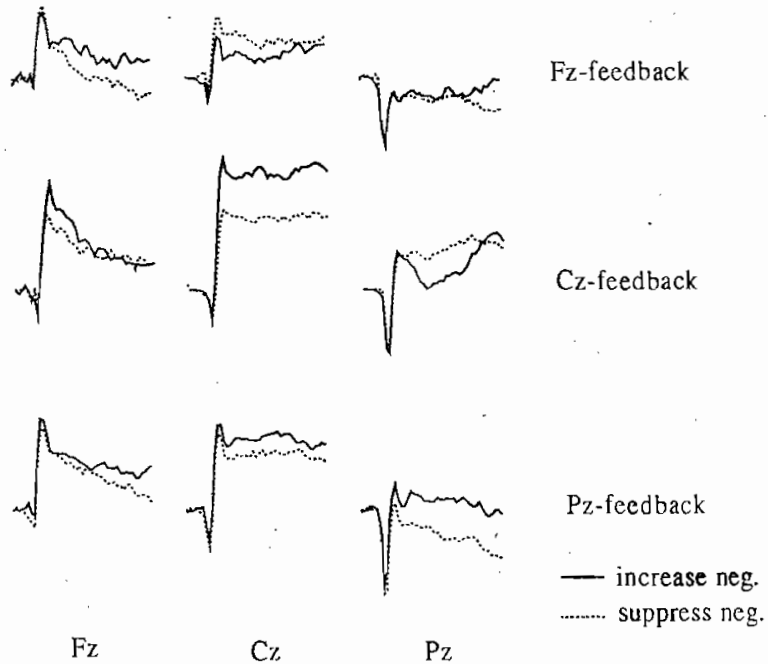

Feedback $\quad-\frac{}{8 \mathrm{~s}}\left[_{+5}^{-5}\right.$

Fig. 1. Slow potential responses on negativity and positivity triais on the third session of area-specific training (last feedback-only session). Siow potentials recorded from $\mathrm{Fz}, \mathrm{Cz}$, and $\mathrm{Pz}$ electrodes (abscissa) are shown for groups receiving feedback at the different midsagittal sites (ordinate).

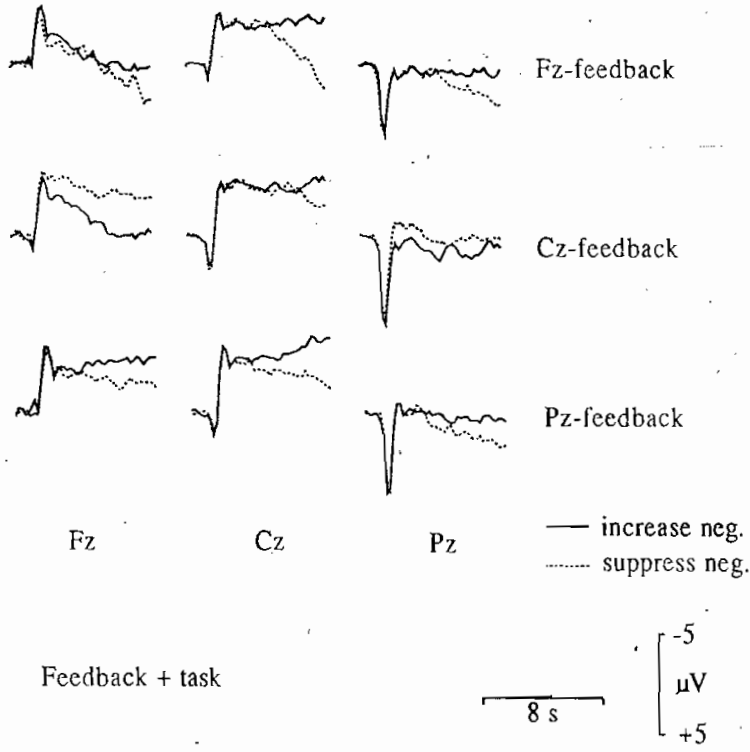

Fig. 2. Slow potential responses when the behavioral tasks were added to the feedback requirement (dual task procedure). Data are presented as in Fig. 1

back trials and self-initiated button-pressing on transfer trials). The SP shifts recorded during this session are shown in Fig. 2, which is arranged identically to Fig. 1. None of the groups successfully differentiated their SPs at any scalp location in the dual-task session $(F$ ratios $<1$ for main effects and interactions attributable to Negativity/Positivity). Impairment of feedback regulation by the dual-task requirement was confirmed when bidirectional differences that were obtained for each subject at the feedback site in session 4 were subtracted from the corresponding bidirectional differences obtained in session 3. Bidirectional performance was poorer on feedback trials of the dual-task session, $t(17)=2.76,. P<0.01$, and also when feedback and transfer trials were combined, $t(17)=$ $2.0, P<0.05$. The deleterious effect of the dual-task requirement did not differ between the feedback groups, indicating a comparable degree of impairment in each feedback condition.

\section{Behavioral tasks}

All groups were given the button-pressing and object-repositioning tasks during the dual-task session. Performance on the task of object-repositioning was measured as the sum of distances between the presented objects and the repositioned ones. These values, and also the number of button presses, were transformed to $z$-scores and assessed by an analysis of variance that included the variables of Group, Negativity/Positivity, and Task (button-pressing versus object repositioning). No significant effects were found, although the interaction of Groups $\times$ Tasks approached significance, $F(2,15)=3.5, P<0.06$. This result re- 
flected a tendency for parietal subjects to press the button more frequently and to reposition objects less accurately than subjects in the frontal group. In neither group did performance vary as a function of whether SP negativity or positivity was signalled during task trials.

\section{Verbal reports}

Although the behavioral tasks were not sensitive to feedback manipulation, the ratings that subjects gave on scales addressing their SP control strategies were affected by the site and polarity of feedback. These ratings are presented in Table I, for negativity and positivity trials separately. Bidirectional differences are not shown in the table but are computable from the results presented there.

Inspection of Table I shows that subjects in the frontal group reported increased concentration, tensing of muscles, and attention to the direction of the rocket when negative SPs were trained (scales 1, 3 and 7, respectively), whereas decreased muscle tension, movement preparation, and aversive emotion were reported when positive SP shifts were required (scales 3, 5 and 6 respectively). Bidirectional differences favoring greater behavioral activation on negativity trials were found for muscle tension, $t(4)=4.71, P<0.01$, preparation for movement, $t(4)=5.73, P<0.01$, concentration, $t(4)=3.26, P<0.05$, and aversive emotion, $t$ (4) $=3.09, P<0.05$, in the frontal group. In contrast to frontal subjects, subjects in the central group tended to report greater behavioral activation on positivity trials than on negativity trials. A bidirectional difference favoring positivity trials was found in reported emotional tension (scale 8), $t(5)=-3.5, P<0.025$, while verbal reports of deep, fast breathing (scale 2) approached bidirectional significance, $t(5)=-2.2, P<$ 0.07 . Parietal subjects, on the other hand, appeared to be behaviorally less active than subjects in the central and frontal groups. Increased concentration (scale 1) and attention to the rocket (scale 7) were reported on negativity trials, and decreased thinking about objects in the room and the visual display (scales 10 and 12) on negativity and positivity trials. Changes in reported muscle tension and movement were not significant in this group (scales 3 and 5, decreases recorded on both trial types). No bidirectional difference reached 2-tailed significance in parietal subjects, but the predicted bidirectional difference in concentration was significant by a 1 -tailed test, $t(4)=2.41, P<0.05$.

Differences in feedback strategy among the frontal, central, and parietal groups were evaluated by applying an analysis of variance to bidirectional differences on the scales of Table I. A main effect of Group was found on 5 scales, these scales assessing deep, fast breathing (scale 2$), F(2,13)=5.41, P<0.02$; preparation for rapid movement (scale 5), $F(2,13)=5.98$, $P<0.02$; emotional tension (scale 8$), F(2,13)=5.89$, $P<0.02$; attention to the rocket (scale 7), $F(2,13)=$ 4.46, $P<0.05$, and to feedback strategy (scale 9$), F(2$, $13)=3.8, P<0.05$. Because these results appeared to reflect predominantly negative bidirectional differences in the central group compared to positive differences in the frontal and parietal groups, $t$ tests were used to contrast the frontal and parietal groups directly. A difference was found on the scale assessing verbal reports of preparation for rapid movement, $t$ $(13)=2.3, \quad P<0.05$. Verbal reports of movement preparation differed bidirectionally in frontal subjects

\section{TABLE I}

Verbal report scales in the frontal, central and parietal groups.

\begin{tabular}{|c|c|c|c|c|c|c|}
\hline \multirow[t]{3}{*}{ Scale } & \multicolumn{6}{|l|}{ Group } \\
\hline & \multicolumn{2}{|l|}{ Frontal } & \multicolumn{2}{|l|}{ Central } & \multicolumn{2}{|l|}{ Parietal } \\
\hline & $\mathrm{Neg}$ & Pos & $\mathrm{Neg}$ & Pos & $\mathrm{Neg}$ & Pos \\
\hline 1. Concentration & $2.4^{* *}$ & -1.2 & 0.7 & 1.2 & $2.8 * *$ & 0.2 \\
\hline 2. Deep fast breath & 0.6 & -1.6 & -1.2 & 1.0 & 0.0 & -1.4 \\
\hline 3. Tense muscles & $1.2 *$ & $-1.2 *$ & -0.5 & -0.5 & -0.2 & -0.8 \\
\hline 4. Imagery & 1.4 & -1.0 & -0.7 & 0.8 & 0.0 & 0.6 \\
\hline 5. Prepare rapid movement & 1.2 & $-2.6 * *$ & -1.7 & -0.2 & -0.8 & -0.8 \\
\hline 6. Aversive emotion & 0.8 & $-2.0^{*}$ & -0.7 & 1.2 & 0.0 & -0.4 \\
\hline 7. Direction of rocket & $2.0 *$ & 1.0 & 0.7 & $2.3 * *$ & $1.8 *$ & 1.0 \\
\hline 8. Emotionally tense & 1.4 & -0.4 & -1.0 & $2.0 * *$ & 1.6 & 0.2 \\
\hline 9. Thought of strategy & 0.8 & 0.2 & 0.0 & 1.5 & 1.0 & -1.2 \\
\hline 10. Objects in room & -0.6 & -0.2 & $-2.2 *$ & -0.7 & $-2.0^{*}$ & -1.4 \\
\hline 11. Stop breathing & 0.6 & -0.6 & -0.8 & -1.3 & 0.2 & -1.0 \\
\hline 12. Objects on screen & 1.0 & -0.4 & -1.0 & -0.7 & $-2.4 * *$ & $-2.4 * *$ \\
\hline
\end{tabular}

Note: entries are mean ratings on each response scale, separately for negativity and positivity trials. A maximum score of +3 (increase) or - 3 (decrease) was possible on each scale $(0=$ midpoint or no change).

* $P<0.05 ; * * P<0.01$ (2-tailed $t$ tests). 
$(P<0.01$, reported above), but not in parietal subjects. $^{3}$

Subjects in the central group reported positivity trials to be more difficult than negativity trials, $t(5)=$ $3.58, P<0.01$. A non-significant difference in the same direction was reported by subjects in the frontal group, and in the opposite direction by parietal subjects. A main effect of group was found on the difficulty measure, $F(2,13)=4.8, P<0.05$, but only when the central group was included in the analysis. Win and loss points complemented this picture. Net earnings were smaller on positivity than on negativity trials in the central group, $t(5)=-4.79, P<0.01$. Frontal subjects earned more win points on negativity than on positivity trials (23.0 and 14.2 win points respectively), while the reverse was true of partietal subjects (14.3 and 20.5 win points respectively), $F(1,10)=6.58$, $P<0.05$. However, loss points and net earnings (winloss) did not differ between the frontal and parietal groups, or between negativity and positivity trials, when the central group was omitted from the analysis.

\section{Discussion}

The present study was undertaken to determine whether SP shifts could be confined to sites along the sagittal midline, when subjects received exteroceptive feedback for area-specific modulation at these sites. Fig. 1 showed that such control was observed after 3 days of feedback training. On the concluding feedback-only session, subjects in the frontal, central, and parietal groups successfully differentiated their SPs between negativity and positivity trials, but only at the feedback electrode (interaction of Groups $X$ Negativity/Positivity $\times$ Electrode site $P<0.05$, SPs referenced to linked ears). Self-regulation did not differ between feedback and transfer trials of this session, indicating that area-specific control of SPs was not feedback dependent.

A further goal of the study was to contrast frontal and parietal SPs with regard to their dependence on motoric functions. Analysis of verbal reports that subjects gave of their control strategies confirmed that different activities were used by frontal and parietal subjects to modulate their SPs. As was expected from previous evidence linking SP negativity with motor performance (Gaillard 1986; Roberts et al. 1989), verbal reports of increased muscle tension and preparation for movement were associated with the production of SP negativity but not positivity in the frontal group (scales 3 and 5 of Table I, bidirectional difference $P<0.01$ in each case). Increased concentration and aversive emotion were also reported on negativity compared to positivity trials in the frontal condition $(P<$ 0.05 ). In contrast, parietal subjects reported response activities of a non-motoric, cognitive nature. Increases in concentration and attention to the rocket were reported on negativity trials $(P<0.05$ or better), and decreases in attention to objects on the screen on negativity as well as positivity trials $(P<0.01)$. Verbal reports of concentration differed bidirectionally in the parietal group ( $P<0.05,1$-tailed). Motoric quiescence, on the other hand, was indicated by non-significant decreases in reported muscle tension and movement preparation on negativity as well as positivity trials, and by non-significant bidirectional differences on scales assessing these activities in parietal subjects. Bidirectional manipulation of movement preparation was found to be significantly greater in the frontal group than in the parietal group, when the groups were compared directly $(P<0.05)$. Thus, in contrast to selfregulation of frontal SPs which was dependent at least in part on motoric generators, self-regulation of parietal SPs did not appear to arise from this source but from attention-like mechanisms. ${ }^{4}$

It may be noteworthy that the properties of feedback-induced parietal negativity resemble those of negativities that have been documented in the stimuluspreceding negativity paradigm. As was true of the parietal feedback case, slow negativities observed in the stimulus-preceding-negativity paradigm appear (i) to relate to information extraction (Ruchkin et al. 1986), (ii) are recorded at post-central sites (Chwilla and Brunia 1991), and (iii) are observed when processes related to response preparation and execution are either removed experimentally (Weinberg 1972; Damen and Brunia 1987) or their effects subtracted out of the resultant wave forms (Damen and Brunia 1991). However, the present results go further and suggest that parietal negativity is not an inevitable concomitant of attention. Differentiation of parietal SPs did not occur in frontal subjects of the present study, even though the verbal reports of these subjects

\footnotetext{
${ }_{3}$ All effects reported above for the verbal reports scales were corroborated at the stated level of significance or better, when non-parametric Wilcoxon and Kruskal-Wallis tests were substituted for parametric $t \mathrm{~s}$ and $F$ s. Because Wilcoxon and Kruskal-Wallis tests can be biased toward rejection of the null hypothesis through elimination of zero difference scores (Wike 1971), we adopted the more conservative parametric procedure (cf., Roberts et al. 1989).
}

\footnotetext{
${ }^{4}$ Although differentiation of verbal report scales addressing motoric functions pointed to a frontal source for SPs modulated in the $\mathrm{Fz}$ group, SPs recorded frontally may also include a component that is volume conducted from the supratemporal plane (see Lutzenberger et al. 1987). Modulation of temporal functions might account for the finding that aversive emotion was also related to slow wave changes in the frontal condition.
} 
alluded to a bidirectional manipulation of attention and sensorimotor performance on negativity compared to positivity trials. These observations imply that slow negativities reflect a "tuning" of neural networks that is distributed across the brain in a highly specific fashion, in accordance with the properties of the task that is presented (Birbaumer et al. 1990). Elbert and Rockstroh (1987) have proposed that tuning is achieved when the polarization of apical dendrites is modulated by cortical reafference acting through thalamic gates. If it is assumed that tuning functions depend on the temporal relation that is experienced among task events (Rockstroh and Elbert 1990; Roberts et al. 1991), SP modulation could an important source of behavioral adaptation in many situations including associative learning.

The results of the central $(\mathrm{Cz})$ group in this study are of interest in relation to previous studies of how SPs at this site are affected by feedback manipulation. Roberts et al. (1989) collected verbal reports after subjects had been trained to modulate SPs at $\mathrm{Cz}$ referred to linked ear lobes (area-non-specific regulation). An offset voltage was included in the feedback algorithm to compensate for onset negativity, following standard practice (Birbaumer et al. 1980). Under these conditions subjects gave verbal reports of muscle tension and movement preparation preferentially on negativity trials $(P<0.01)$, which was true also of subjects trained frontally in the present experiment. These findings are consistent with the known representation of sensorimotor functions in the cortical tissues proximal to these scalp electrodes. However, in the present study in which an offset voltage was omitted, a different result was obtained in central subjects. Because the negative SP shift elicited by visual stimulation was pronounced at $\mathrm{Cz}$ (Fig. 1), central subjects achieved success with little effort on negativity trials, whereas on positivity trials extensive error feedback was encountered. Hence $\mathrm{Cz}$ subjects rated positivity trials to be more difficult than negativity trials, lost more bonus points on positivity trials, and gave verbal reports of behavioral activation when positivity was called for. It is noteworthy that the two verbal report scales that showed the largest bidirectional differences in $\mathrm{Cz}$ subjects were those assessing emotional tension $(P<0.025)$ and fast, deep breathing $(P<0.07)$, both differences favoring SP positivity. Elbert et al. (1992) found bidirectional differences $(P<0.02)$ on the same two verbal report scales, when feedback was given for increases and decreases in blood pressure rather than for SPs. Emotional arousal and deep, fast breathing were associated with blood pressure increases in the Elbert et al. (1992) study, and produced a positive-going SP shift at $\mathrm{Cz}$, apparently by activating the baroreceptors.

Behavioral tasks (button-pressing and object-repositioning) were added in the fourth session of the pres- ent experiment, to further probe the functional significance of self-regulated frontal and parietal SPs. However, this dual-task approach did not work, because self-regulation of SPs (area-specific and otherwise) failed when the behavioral tasks were concurrently presented. This evidence for task interference indicates that feedback regulated electrocortical responses are not readily performed outside of attention (cf., Black and Cott 1977). Why task interference was more apparent in the present study than in earlier experiments in which area-non-specific SP regulation was trained is not clear (see Rockstroh et al. 1989, for a review of the earlier studies). Relevant variables may include the difficulty of the area-specific feedback problem, the particular cortical networks that were regulated, and/or the behavioral tasks applied. However, it should be noted that modulation of task performance in earlier research was typically not evaluated with respect to single-task baselines, as was done in the present experiment (dual-task session 4 compared to feedback-alone session 3). It is therefore possible that modulation of task performance by SP shifts in earlier studies was caused more by a suppression of task performance consequent on one polarity of SP shift, than by an enhancement of task performance when the opposite SP shift was produced.

\section{References}

Birbaumer, N., Elbert, T., Rockstroh, B. and Lutzenberger, W. Biofeedback of event-related slow potentials of the brain. Int. J. Psychol., 1980, 16: 389-415.

Birbaumer, N., Lang, P., Cook, E., Elbert, T., Lutzenberger, W. and Rockstroh, B. Slow brain potentials, imagery and hemispheric differences. Int. J. Neurosci., 1988, 39: 101-116.

Birbaumer, N., Elbert, T., Canavan, A. and Rockstroh, B. Slow potentials of the cerebral cortex and behavior. Physiol. Rev., 1990, 70: 1-41.

Black, A.H. and Cott, A. The operant learning theory approach to biofeedback training. In: G.E. Schwartz and J. Beatty (Eds.), Biofeedback: Theory and Research. Academic Press, New York, 1977: 89-127.

Chwilla, D.J. and Brunia, C.H.M. Event-related potentials to different feedback stimuli. Psychophysiology, 1991, 28: 123-132.

Coles, M.G.H. Modern mind-brain reading: psychophysiology, physiology and cognition. Psychophysiology, 1989, 26: 251-269.

Damen, E.J.P. and Brunia, C.H.M. Changes in heart rate and slow brain potentiais related to motor preparation and stimulus anticipation in a time estimation task. Psychophysiology, 1987, 24: $700-713$.

Damen, E.J.P. and Brunia, C.H.M. Subtracting pre- and post-movement potentials reveals the true stimulus preceding negativity. Psychophysiology, 1991, 28: S18.

Elbert, T. and Rockstroh, B. Some remarks on the development of a standardized time constant. Psychophysiology, 1980, 17: 504-505.

Elbert, T. and Rockstroh, B. Threshold regulation - a key to the understanding of the combined dynamics of EEG and event-related potentials. J. Psychophysiol., 1987, 1: 317-333.

Elbert, T., Lutzenberger, W., Rockstroh, B. and Birbaumer, N. Removal of ocular artifacts from the EEG: a biophysical ap- 
proach to the EOG. Electroenceph. clin. Neurophysiol., 1985, 60: 455-463.

Elbert, T., Roberts, L., Lutzenberger, W. and Birbaumer, N. Modubation of slow cortical potentials by instrumentally learned blood pressure responses. Psychophysiology, 1992, in press.

Gaillard, A.W.K. The CNV as an index of response preparation. In: - w.C. McCallum, R. Zappoli and F. Denoth (Eds.), Cerebral Psychophysiology: Studies in Event Related Potentials. Electroenceph. clin. Neurophysiol., Suppl. 38. Elsevier, Amsterdam, 1986: 196-206.

Lutzenberger, W., Elbert, T. and Rockstroh, B. A brief tutorial on the implications of volume conduction for the interpretation of the EEG. J. Psychophysiol., 1987, 1: 81-89.

Roberts, L.E., Williams, R.J., Marlin, R.G., Farrell, T. and Imiolo, $D$. Awareness of the response after feedback training for changes in heart rate and sudomotor laterality. J. Exp. Psychol.: Gen., 1984, 113: 225-255.

Roberts, L.E., Birbaumer, N., Rockstroh, B., Lutzenberger, W. and Elbert, T. Self-report during feedback regulation of slow cortical potentials. Psychophysiology, 1989, 26: 392-403.

Roberts, L.E., Preston, D. and Uttl, B. Tuning and capacity limitations in feedback (instrumental) learning. Paper presented at the annual meetings of the Psychonomic Society, San Francisco, CA, 1991.

Rockstroh, B. and Elbert, T. On the regulation of excitability in cerebral cortex - a bridge between EEG and attention? In: H.G. Geissler (Ed.), Psychophysical Explorations of Mental Structures. Hogrefe and Huber, Toronto, 1990: 323-332.

Rockstroh, B., Elbert, T., Lutzenberger, W. and Birbaumer, N. The effects of slow cortical potentials on response speed. Psychophysiology, 1982, 19: 211-217.
Rockstroh, B., Birbaumer, N., Elbert, T. and Lutzenberger, W. Operant control of EEG and event-related and slow brain potentials. Biofeed. Self-Regul., 1984, 9: 139-160.

Rockstroh, B., Elbert, T., Canavan, A., Lutzenberger, W. and Birbaumer, N. Slow Cortical Potentials and Behavior. Urban and Schwarzenberg, Munich, 1989.

Rockstroh, B., Elbert, T., Lutzenberger, W. and Birbaumer, N. Biofeedback: evaluation and therapy in children with attentional dysfunctions. In: A. Rothenberg (Ed.), Brain and Behavior in Child Psychiatry. Springer, Berlin, 1990a: 345-357

Rockstroh, B., Elbert, T., Birbaumer, N. and Lutzenberger, W. Biofeedback-produced hemispheric asymmetry of slow cortical potentials and its behavioural effects. Int. J. Psychophysiol., 1990b, 9: $151-165$.

Rösler, F. and Heil, M. Toward a functional categorization of slow waves: taking into account past and future events. Psychophysiology, 1991, 28: 344-358.

Ruchkin, D.S., Sutton, S., Mahaffey, D. and Glaser, J. Terminal CNV in the absence of motor response. Electroenceph. clin. Neurophysiol., 1986, 63: 445-463.

Stamm, J. Performance enhancements with cortical negative slow potential shifts in monkey and man. In: T. Elbert, B. Rockstroh, W. Lutzenberger and N. Birbaumer (Eds.), Self-Regulation of the Brain and Behavior. Springer, Heidelberg, 1984: 199-215.

Tecce, J. Contingent negative variation (CNV) and psychological processes in man. Psychol. Bull., 1972, 77: 72-108.

Weinberg, H. The contingent negative variation: its relation to feedback and expectant attention. Neuropsychologia, 1972, 10: 299-306.

Wike, E.L. Data Analysis. Aldine Atherton, Chicago, IL, 1971. 